\title{
Infective Endocarditis as a Complication of Intravenous Drug Use in Pregnancy: A Retrospective Case Series and Literature Review
}

\author{
Adebayo Adesomo, MD ${ }^{1}$ Veronica Gonzalez-Brown, MD ${ }^{1}$ Kara M. Rood, MD ${ }^{1}$ \\ ${ }^{1}$ Department of Obstetrics and Gynecology, The Ohio State \\ University College of Medicine, Columbus, Ohio \\ Address for correspondence Adebayo Adesomo, MD, Department of \\ Obstetrics and Gynecology, The Ohio State University, Columbus, \\ Am J Perinatol Rep 2020;10:e288-e293. \\ OH 43210 (e-mail: adebayo.adesomo@osumc.edu).
}

\begin{abstract}
Objective An increase in opioid use disorder and subsequent intravenous drug use has led to an increase in sequalae that may complicate pregnancy, such as infective endocarditis. Infective endocarditis has the potential for significant maternal and neonatal morbidity and mortality. We sought to examine the management considerations and clinical implications of intravenous drug use-related infective endocarditis in pregnancy from our center's experience.

Study Design Retrospective study of management of pregnancies complicated by infective endocarditis as a result of active intravenous drug use at an academic tertiary care hospital from January 2012 through December 2019.

Results Twelve women with active intravenous drug use histories were identified as having clinical and echocardiographic features consistent with infective endocarditis. Six women were discharged against medical advice and did not complete the full course of recommended antibiotic regimen. Eight women were started or continued on opioid

\section{Keywords}

- infective endocarditis

- pregnancy

- intravenous drug use

- bacteremia

- opioid use disorder agonist therapy during their hospitalization. Four neonates required neonatal intensive care unit admission for pharmacologic treatment for neonatal abstinence syndrome. Conclusion Management of intravenous drug use-associated infective endocarditis in pregnancy involves more than treating the acute condition. In pregnant women with opioid use disorder and infective endocarditis, addiction and chronic psychosocial conditions need to be addressed to optimize care.
\end{abstract}

Opioid use disorder (OUD) and its associated complications in the reproductive age population are reaching epidemic proportions. ${ }^{1,2}$ As rates of OUD continue to rise, the incidence of pregnancy-related sequalae of OUD such as peripartum maternal opioid overdoses, neonatal abstinence syndrome, and hepatitis $C$ infections has also increased. ${ }^{3}$ The incidence of infective endocarditis (IE), a complication of OUD most commonly associated with intravenous drug use (IDU), is also increasing. ${ }^{10,11}$ Due to a historically low incidence, literature on IE in pregnancy is mostly limited to individual case reports and systematic reviews. ${ }^{12}$ Although treatment of IE in pregnancy is extrapolated from general population research, there

received

March 20, 2020

accepted

June 4, 2020
DOI https://doi.org/

$10.1055 / \mathrm{s}-0040-1716732$. ISSN 2157-6998. are added complexities of treating IE associated with IDU in the antenatal or perinatal periods that require special attention. ${ }^{13-15}$ In addition to the acute treatment of IE, treatment for addiction, support for chronic psychosocial conditions, and knowledge of state-specific medicolegal environments may be needed to optimize outcomes. Given the current opioid epidemic, we noted an increase in pregnancy-associated admissions for IE stemming from IDU at our institution. As an institution with a dedicated Substance Abuse Treatment and Prevention Program for opioid dependent pregnancies, our objective is to add to the existing literature on the management of IE resulting from IDU in pregnancy and highlight
Copyright $\odot 2020$ by Thieme Medical

Publishers, Inc., 333 Seventh Avenue, New York, NY 10001, USA. Tel: $+1(212) 760-0888$
License terms

(요 (1) $\Theta$ 
important aspects of antepartum care for IE as they relate to OUD and IDU.

\section{Methods}

In this retrospective cohort case series, we identified women with a diagnosis of IE related to IDU during a pregnancy episode from years 2012 to 2019. A search of electronic medical records for pregnancy episodes with the associated diagnosis of IE was conducted. International Classification of Diseases-9th revision/10th revision (ICD-9/ICD-10) diagnostic codes for diseases of the circulatory system and IE complicating pregnancy were used for the search. In addition, combinations of keywords such as "infective endocarditis," "opioid use disorder," and "polysubstance abuse" were used to search the Epic Integrated Health Information Systems patient "Problem List." The "Problem List" tracks active and historical diagnoses entered into the patient's charts by providers. A total of 57 patients were identified. This initial cohort was screened for IE during an active pregnancy episode by using Duke's modified criteria, namely the presence of positive blood cultures and echocardiographic findings consistent with endocardial vegetations. Women who were not persons who inject drugs or who did not have acute IE during a pregnancy episode (antepartum or within 4 weeks postpartum) were excluded ( - Fig. 1-create flow diagram)

The medical records of the 12 women who met inclusion criteria were reviewed. Data on demographics, past medical and obstetrical histories, drug use history, details of antepartum hospital admissions and treatment courses for IE, echocardiographic data, initiation of opioid agonist therapy or medication-assisted treatment, prenatal outpatient follow-up, pregnancy outcomes including delivery complications and neonatal outcomes, postpartum follow-up, and hospital readmissions in the peripartum period were collected. Descriptive statistics (mean \pm standard deviation for normally distributed continuous variables and median and interquartile range for non-normally distributed continuous variables; frequencies and percentages for categorical variables) were calculated. This study was approved by The Ohio State University Institutional Review Board.

\section{Results}

\section{Patient Characteristics}

The 12 women within our cohort had a mean age of 30 $( \pm 4.58)$. The majority was white $(80 \%)$ (-Table 1$)$. All were known to be persons who inject drugs and the majority (67\%) received prenatal care through our substance abuse specialty prenatal clinic. The vast majority had a coexisting diagnosis of hepatitis C (92\%). Nine women were treated for endocarditis in the antepartum period and two within 4 weeks of the postpartum period. One patient presented for new obstetric care in the setting of incompletely treated endocarditis prior to conception. One patient was discharged without antibiotic therapy after additional imaging proved her cardiac lesion to be more consistent with previously treated IE. The majority of women (84\%) presented with cardiopulmonary symptoms including shortness of breath, dyspnea on exertion, chest pain with or without fever. The remainder presented with symptoms related to the infectious sequalae of IDU, including extremity pain from injection site, abscess, and hip pain secondary to sacroiliitis (16\%).

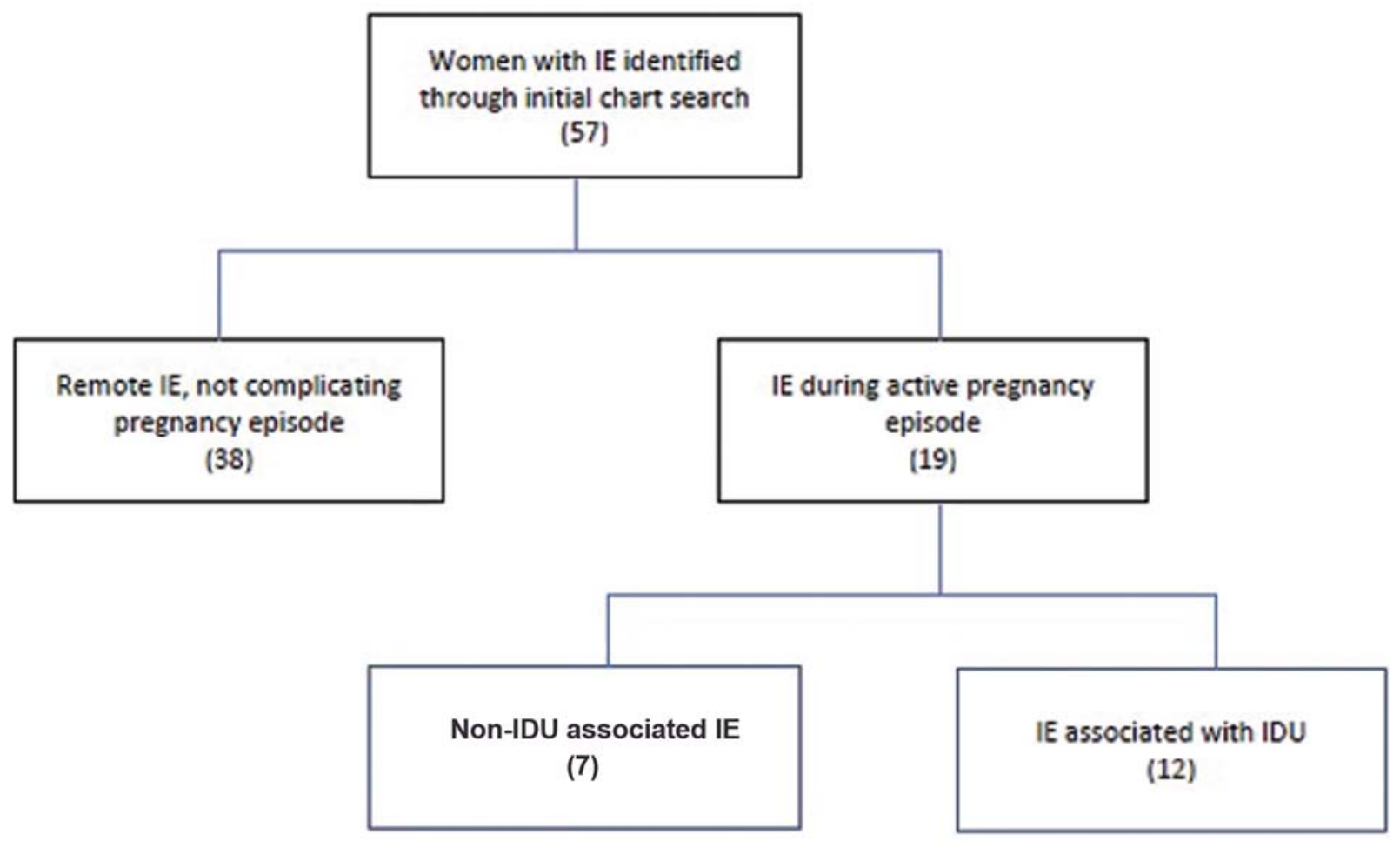

Fig. 1 Patient selection flowchart. IDU, intravenous drug use; IE, infective endocarditis. 
Table 1 Demographic and clinical characteristics of patients with IE

\begin{tabular}{|c|c|}
\hline Characteristics & \\
\hline Age $(y)$, mean $( \pm S D)$ & $30.2 \pm 4.58$ \\
\hline $\begin{array}{l}\text { Gestational age at diagnosis (wk), } \\
\text { mean (IQR) }\end{array}$ & $25.4(21-31)$ \\
\hline \multicolumn{2}{|l|}{ Caucasian } \\
\hline Antepartum diagnoses $(n)$ & $10(83 \%)$ \\
\hline Postpartum diagnoses $(n)$ & $2(17 \%)$ \\
\hline Comorbidities & $n(\%)$ \\
\hline Hepatitis C & $11(92)$ \\
\hline Psychiatric disorder $^{a}$ & $3(25)$ \\
\hline Valve involvement & $n(\%)$ \\
\hline Native valve & $11(92)$ \\
\hline Prosthetic & $1(8)$ \\
\hline Left sided, single & $2(17)$ \\
\hline Mitral & $2(100)$ \\
\hline Aortic & $0(0)$ \\
\hline Right sided, single & $8(66)$ \\
\hline Tricuspid & $7(86)$ \\
\hline Pulmonic & $1(14)$ \\
\hline $\begin{array}{l}\text { Multivalvular } \\
\text { (both sides) }\end{array}$ & $2(17)$ \\
\hline
\end{tabular}

Abbreviations: IE, infective endocarditis; IQR, interquartile range; SD, standard deviation.

aPsychiatric disorders including depression, anxiety, bipolar disorder.

\section{Echocardiographic Findings}

Echocardiograph results were available for all women in our cohort (-Table 2 ). The majority of these studies was transthoracic versus transesophageal echocardiograms (91\%). Discrete vegetations were visualized in $80 \%$ of the patients, mostly commonly involving the tricuspid valve (56\%). When measured quantitatively, valvular lesions ranged from $0.6 \times 0.8 \mathrm{~cm}$ to $3.0 \times 1.6 \mathrm{~cm}$ in size. Otherwise, lesions were classified as either small or large in echocardiographic reports. Interval echocardiographs were available for six women and the majority of these (83\%) had interval decrease in vegetation size after antibiotic treatment.

\section{Blood Cultures and Antibiotic Therapy}

Bacteremia with Staphylococcal aureus was identified in three-fourths of the women (75\%). Vancomycin was the most commonly used antibiotic (58\%); however, transition from vancomycin due to side effects occurred in five cases. Daptomycin was the most frequently used alternative, but ceftaroline fosamil and linezolid were also used. Recommended antibiotic course lengths ranged from 2 to 8 weeks. Approximately half of these women did not complete their recommended antibiotic course due to discharge against medical advice (AMA). Circumstances surrounding discharge AMA were not consistently specified in the medical records.
Table 2 Microbiologic and treatment data

\begin{tabular}{|c|c|}
\hline & $n(\%)$ \\
\hline \multicolumn{2}{|l|}{ Bacteria } \\
\hline Methicillin-resistant Staphylococcus aureus & $5(42)$ \\
\hline Methicillin-sensitive Staphylococcus aureus & $4(33)$ \\
\hline Beta-hemolytic streptococcus, Group C & $1(8)$ \\
\hline None & $2(17)$ \\
\hline \multicolumn{2}{|l|}{ Treatment } \\
\hline Antibiotic regimen complete & $6(60)$ \\
\hline Length of hospital stay (median, IQR) & $26(16-39)$ \\
\hline Resolution of vegetation & $4(66)$ \\
\hline
\end{tabular}

Abbreviation: IQR, interquartile range.

Reasons cited included continued illicit drug use while inpatient (e.g., crushing oral opioids for self-injection) and the reported need to handle family affairs outside of the hospital setting.

\section{Opioid Use Disorder}

All women were known to be persons who inject drugs and the majority (67\%) received prenatal care through our substance abuse specialty prenatal clinic. Urine toxicology report revealed that all women had tested positive for opioids either upon admission or in the recent past preceding current admission. Other nonprescribed, illicit substances discovered in toxicology reports included cocaine, amphetamines, tetrahydrocannabinol, and benzodiazepines. During the time of admission, the majority of women (92\%) received treatment for OUD through either the initiation or continuation of opioid agonist and antagonist medications such as suboxone (67\%), methadone (17\%), or Subutex (1\%) (-Table 3). Inpatient psychosocial interventions were routinely offered to all women in conjunction with psychiatric and social work consultation services. Additionally, all women had the option of continuing prenatal care through our substance abuse specialty prenatal clinic where peer support is available through group prenatal care.

\section{Outcomes}

Median gestational age at diagnosis was 25 weeks (interquartile range [IQR] 21-31) and median gestational age at delivery was 37 weeks (35-38). There were four cesarean deliveries (33\%) for usual obstetrical indications. The median duration of hospital course was 26 days (16-39). Besides OUD and hepatitis C virus, septic pulmonary emboli (73\%), anemia of chronic disease (55\%), and acute kidney injury (45\%) were the most commonly encountered comorbidities. There was one maternal death, after urgent cardiac surgery for heart failure secondary to severe aortic and tricuspid valve vegetations and ventricular septum abscess. The remaining patients had clinical improvement or interval improvement of echocardiographic findings. Sixty-seven percent of patients were initiated on medication-assisted 
Table 3 Opioid use disorder data

\begin{tabular}{|c|c|}
\hline & $n(\%)$ \\
\hline \multicolumn{2}{|l|}{ Urine toxicology results } \\
\hline Opioids (heroin or prescription) & $12(100)$ \\
\hline Cocaine & $5(42)$ \\
\hline Amphetamines & $3(25)$ \\
\hline THC & $3(25)$ \\
\hline Benzodiazepines & $3(25)$ \\
\hline \multicolumn{2}{|l|}{ Treatment of OUD during admission } \\
\hline Suboxone & $8(67)$ \\
\hline Methadone & $2(17)$ \\
\hline Subutex & $1(8)$ \\
\hline \multicolumn{2}{|l|}{ Neonatal abstinence syndrome } \\
\hline NICU admission & $4(44)$ \\
\hline
\end{tabular}

Abbreviations: NICU, neonatal intensive care unit; THC, tetrahydrocannabinol; OUD, opioid use disorder.

treatment during their hospitalization and were continued on treatment in our OUD program.

Over half of pregnancies delivered at term (55\%) and the majority of preterm deliveries was iatrogenic (75\%) for reasons unrelated to OUD or IE. The one case of maternal cardiac surgery that required extracorporeal membrane oxygenation resulted in intrauterine fetal demise at 19 weeks gestation. There was one case of a term fetal demise at 38 weeks gestation as a result of placental abruption. Four of the neonates (44\%) required neonatal intensive care unit admission and pharmacologic treatment for neonatal abstinence syndrome of varying severity. All living newborns were discharged between day of life 4 and 25 days (median $=12,[5-16]$ ).

The majority of patients was lost to cardiothoracic surgery follow-up (64\%); however, none presented for recurrent IE within our 4 weeks follow-up period. Thus, a third did have subsequent clinical encounters stemming from continued IDU (33\%).

\section{Discussion}

IE during pregnancy is rare with a reported incidence of $0.006 \%{ }^{13,16} \mathrm{~A}$ recent systematic review of peripartum IE reported maternal and fetal mortality rates of 11 and 14\%; these rates have traditionally been higher, up to 33 and $29 \%$ for mother and fetus, respectively. ${ }^{13,17,18}$ The pathogenic mechanism of IE in IDU involves endothelial damage to cardiac valves from intravascular injection of foreign particulate matter while using illicit drugs. ${ }^{19}$ In a similar manner, bacterial contaminant from skin or contaminated syringes may also be inadvertently injected into the blood stream during drug usage. ${ }^{20}$

IE should be suspected in any patient presenting with a fever and a new onset heart murmur; the clinical and echocardiographic features of Duke's criteria can be used to formally make the diagnosis. Major diagnostic criteria include positive blood cultures for microorganisms typical of IE and echocardiographic evidence of endocardial involve- ment. ${ }^{13}$ Alternatively, septic pulmonary emboli, pneumonia, or positive blood cultures for common skin contaminants in the context of IDU history may prompt echocardiographic imaging and subsequent diagnoses of IE. ${ }^{15}$

The most commonly identified bacterial pathogens in patients with IE are Staphylococcus aureus and Streptococcus viridians. ${ }^{21,22}$ Courses of up to 8 weeks of intravenous antibiotics may be indicated for methicillin-resistant Staphylococcus aureus, whereas shorter courses may suffice for uncomplicated IE due to isolated methicillin-sensitive Staphylococcal bacteremia. ${ }^{23}$ For cases of acute heart failure or those refractory to medical management, cardiac surgery for valve replacement may be necessary. Cardiac surgery and cardiopulmonary bypass carry significant risk of mortality, up to 30 and $6 \%$ for fetus and mother, respectively. ${ }^{24,25}$ In pregnant women, with nonurgent indications for surgery, the fetal benefits of an advancing gestation must be weighed against the potential of an acutely worsening cardiac status. Literature for cardiac surgery and cardiopulmonary bypass in pregnancy suggests elective delivery prior to maternal cardiac surgery improves fetal outcome at later gestational ages. $^{26}$ In theory, high-flow, high-pulsatile, normothermic CPB may improve uterine blood flow although clinical studies for optimal settings are lacking. ${ }^{27,28}$

Successful antepartum care of patients with IE in pregnancy may be fraught with difficulties stemming from concomitant OUDs, the nature of extended-length hospital stays for the treatment of IE, AMA discharges, and chronic comorbidities. $^{14,29}$ Among persons who use drugs, IDU, in particular, has been implicated as an independent risk factor for discharge AMA. $^{30,31}$ Previous research highlights the negative impact discharge AMA may have for pregnancies in vulnerable patient populations such as women with substance abuse. ${ }^{32}$ In contrast, in-hospital opioid agonist therapy has been demonstrated as a negative risk factor for discharge AMA in this same population. In addition to in-hospital retention, the potential benefits of initiating opioid agonist therapy for hospitalized antepartum patients include improved obstetrical outcomes that are related to the discontinued use of illicit substances and improved prenatal care compliance. ${ }^{33}$

The historical barriers to successful initiation and maintenance of medication-assisted treatment for OUD are manifold. In pregnancy, a lack of screening and under diagnosis predisposed to suboptimal rates of treatment of OUD in antepartum period. ${ }^{34,35}$ Early research controversies suggesting an association between opioid exposure and birth defects, along with concerns regarding risks of neonatal abstinence syndrome, created reservations for the routine prescription of opioid agonists to treat OUD. ${ }^{36,37}$ Lastly, when treatment for OUD was actually pursued, approaches associated with failure (e.g., lack of third trimester up-titration) or high rates of relapse (e.g., medically supervised withdrawal) may have been deployed. $^{38,39}$ Time and research have addressed many of these controversies; however, large proportions of pregnant women still do not receive opioid agonist therapies when hospitalized. ${ }^{40-44}$

State-mandated reporting and the threat of civil or criminal penalties including the loss of child custody hinder the 
physician-patient therapeutic alliance and potentially discourage women from seeking and completing needed therapy for sequelae of OUD such as intravenous drug user (IVDU)associated IE. ${ }^{45-47}$ As of May 2020, 25 states require healthcare providers to report suspected prenatal drug use and 23 states consider such child abuse under civil child-welfare statutes, whereas only 19 states have targeted drug treatment programs for pregnant women. ${ }^{48}$ In addition to advocating for less punitive legislation for drug use in pregnancy, treating obstetrician-gynecologists should be knowledgeable of their individual state's policies and be wary of the role such policies may have in the nonadherence to treatment regimens.

In addition to addressing OUD, healthcare professionals should be aware of the other chronic comorbid conditions that may complicate care during antepartum hospitalizations. Women with OUD have a high prevalence of undiagnosed, chronic mood disorders. Fitzsimons prospectively observed a greater than $70 \%$ rate of co-occurring mood or anxiety disorders for pregnant women entering into a treatment center for substance use disorders. ${ }^{49}$ At equipped hospital facilities, women admitted for the treatment of IVDU-associated IE should be screened for and have management of underlying psychosocial conditions in consultation with appropriate psychiatric and social work services.

Even with optimal management of underlying addiction and comorbid psychiatric conditions, the extended courses needed to complete therapy for IE in the hospital setting may be difficult for any woman. Strategies of motivational interviewing, as well as the utilization of shorter duration treatment courses for uncomplicated IE may improve adherence. Restrictive hospital policies during these admissions (e.g., offward privileges, limited guess access), aimed at reducing the risks of continued, surreptitious drug use, may inadvertently increase rates of discharge AMA. Ultimately, an increase in the availability of long-term acute health facilities that accept pregnant women may be needed. Recent data highlights the importance of women-centered treatment programs in improving pregnancy-specific outcomes such as postpartum visit attendance, breastfeeding continuation rates, and longacting contraceptive utilization. . $^{5,51}$

\section{Conclusion}

IE in pregnancy, as it relates to IDU, may be associated with significant maternal and fetal morbidity. In pregnant patients with OUD and IE, additional considerations must be taken into account to improve outcomes. The initiation of opioid agonist therapy, psychosocial support, and services to address underlying psychiatric diagnoses, improved access to long-term acute health facilities that accept pregnant women, and strategies to minimize AMA discharge and incomplete treatment must all be considered to optimize treatment of this vulnerable population.

\section{Funding}

The authors report no external funding source for this study.

\section{Conflict of Interest}

The authors declare no conflict of interest.

\section{References}

1 Compton WM, Jones CM, Baldwin GT. Relationship between nonmedical prescription-opioid use and heroin use. $\mathrm{N}$ Engl J Med 2016;374(02):154-163

2 Desai RJ, Hernandez-Diaz S, Bateman BT, Huybrechts KF. Increase in prescription opioid use during pregnancy among Medicaidenrolled women. Obstet Gynecol 2014;123(05):997-1002

3 Metz TD, Rovner P, Hoffman MC, Allshouse AA, Beckwith KM, Binswanger IAMaternal Deaths from Suicide and Overdose in Colorado, 2004-2012. In: Obstetrics and Gynecology. Vol 128. Lippincott Williams and Wilkins; 2016:1233-1240. Doi: 10.1097/ AOG.0000000000001695

4 Schiff DM, Nielsen T, Terplan M, et al. Fatal and nonfatal overdose among pregnant and postpartum women in Massachusetts. Obstet Gynecol 2018;132(02):466-474

5 Smid MC, Stone NM, Baksh L, et al. Pregnancy-associated death in Utah: contribution of drug-induced deaths. Obstet Gynecol 2019; 133(06):1131-1140

6 Virginia Department of Health. (2015). Pregnancy-Associated Deaths from Drug Overdose in Virginia, 1999-2007. Richmond: Virginia Department of Health, Office of the Chief Medical Examiner

7 Patrick SW, Davis MM, Lehmann CU, Cooper WO. Increasing incidence and geographic distribution of neonatal abstinence syndrome: United States 2009 to 2012. J Perinatol 2015;35(08):650-655

8 Patrick SW, Schumacher RE, Benneyworth BD, Krans EE, McAllister JM, Davis MM. Neonatal abstinence syndrome and associated health care expenditures: United States, 2000-2009. JAMA 2012; 307(18):1934-1940

9 Rossi RM, Warshak CR. Prevalence of maternal hepatitis C virus infection in Ohio. Obstet Gynecol 2018;132(03):708-716

10 Hartman L, Barnes E, Bachmann L, Schafer K, Lovato J, Files DC. Opiate injection-associated infective endocarditis in the southeastern United States. Am J Med Sci 2016;352(06):603608

11 Jones CM, Christensen A, Gladden RM. Increases in prescription opioid injection abuse among treatment admissions in the United States, 2004-2013. Drug Alcohol Depend 2017;176:89-95

12 Kebed KY, Bishu K, Al Adham RI, et al. Pregnancy and postpartum infective endocarditis: a systematic review. Mayo Clin Proc 2014; 89(08):1143-1152

13 Habib G, Lancellotti P, Antunes MJ, et al. 2015 ESC guidelines for the management of infective endocarditis: the task force for the management of infective endocarditis of the European Society of Cardiology (ESC): endorsed by: European Association for Cardio-thoracic Surgery (EACTS), the European Association of Nuclear Medicine (EANM). Russ J Cardiol 2016;133(05): 65-116

14 Prasad M, Jones M. Medical complications of opioid use disorder in pregnancy. Semin Perinatol 2019;43(03):162-167

15 Baddour LM, Wilson WR, Bayer AS, et al. 2015). Infective endocarditis in adults: Diagnosis, antimicrobial therapy, and management of complications: A scientific statement for healthcare professionals from the American Heart Association. In Circulation (Vol. 132, Issue 15, pp. 1435-1486). Lippincott Williams and Wilkins. https://doi. org/10.1161/CIR.0000000000000296

16 Montoya ME, Karnath BM, Ahmad M. Endocarditis during pregnancy. South Med J 2003;96(11):1156-1157

17 Dajani AS, Taubert KA, Wilson W, et al. Prevention of bacterial endocarditis. Recommendations by the American Heart Association. Circulation 1997;96(01):358-366

18 Birincioglu CL, Unal EU, Çelik IH, et al. Surgery for rheumatic valve disease in pregnancy: what about the newborn? Heart Lung Circ 2014;23(01):63-67 
19 Werdan K, Dietz S, Löffler B, et al. Mechanisms of infective endocarditis: pathogen-host interaction and risk states. Nat Rev Cardiol 2014;11(01):35-50

20 Sande MA, Lee BL, Mills J, et al. Endocarditis in intravenous drug users. In: Kaye D, ed. . Infective Endocarditis. New York: Raven Press; $1992: 345$

21 Murdoch DR, Corey GR, Hoen BInternational Collaboration on Endocarditis-Prospective Cohort Study (ICE-PCS) Investigators. , et al; . Clinical presentation, etiology, and outcome of infective endocarditis in the 21st century: the International Collaboration on EndocarditisProspective Cohort Study. Arch Intern Med 2009;169(05):463-473

22 Selton-Suty C, Célard M, Le Moing VAEPEI Study Group., et al; . Preeminence of Staphylococcus aureus in infective endocarditis: a 1year population-based survey. Clin Infect Dis 2012;54(09):1230-1239

23 Torres-Tortosa M, de Cueto M, Vergara AGrupo de Estudio de Enfermedades Infecciosas de la Provincia de Cádiz. , et al; . Prospective evaluation of a two-week course of intravenous antibiotics in intravenous drug addicts with infective endocarditis. Eur J Clin Microbiol Infect Dis 1994;13(07):559-564

24 Weiss BM, Von Segesser LK, Alon E, Seifert B, Turina MI. Outcome of cardiovascular surgery and pregnancy: a systematic review of the period 1984-1996. In: American Journal of Obstetrics and Gynecology. Vol 179. Mosby Inc.; 1998:1643-1653. Doi: 10.1016/ S0002-9378(98)70039-0

25 Born D, Massonetto JC, de Almeida PA, et al. [Heart surgery with extracorporeal circulation in pregnant women. Analysis of maternofetal outcome]. Arq Bras Cardiol 1995;64(03):207-211http://www. ncbi.nlm.nih.gov/pubmed/7487505 Accessed March 3, 2020

26 John AS, Gurley F, Schaff HV, et al. Cardiopulmonary bypass during pregnancy. Ann Thorac Surg 2011;91(04):1191-1196

27 Sepehripour AH, Lo TT, Shipolini AR, McCormack DJ. Can pregnant women be safely placed on cardiopulmonary bypass? Interact Cardiovasc Thorac Surg 2012;15(06):1063-1070

28 Strickland RA, Oliver WC Jr, Chantigian RC, Ney JA, Danielson GK. Anesthesia, cardiopulmonary bypass, and the pregnant patient. Mayo Clin Proc 1991;66(04):411-429

29 Morita Y, Haruna T, Haruna Y, et al. Thirty-day readmission after infective endocarditis: analysis from a nationwide readmission database. J Am Heart Assoc 2019;8(09):e011598

$30 \mathrm{Ti}$ L, Ti L. Leaving the hospital against medical advice among people who use illicit drugs: a systematic review. Am J Public Health 2015;105(12):e53-e59

31 Chan AC, Palepu A, Guh DP, et al. HIV-positive injection drug users who leave the hospital against medical advice: the mitigating role of methadone and social support. J Acquir Immune Defic Syndr 2004;35(01):56-59

32 Tucker Edmonds B, Ahlberg C, McPherson K, Srinivas S, Lorch S. Predictors and adverse pregnancy outcomes associated with antepartum discharge against medical advice. Matern Child Health J 2014;18(03):640-647

33 Batki SL A Treatment Improvement Protocol Medication-Assisted Treatment For Opioid Addiction in Opioid Treatment Programs TIP 43 A Treatment Improvement Protocol TIP 43 Medication-Assisted Treatment For Opioid Addiction in Opioid Treatment Programswww.samhsa.gov. Accessed May 15, 2020.

34 Garg M, Garrison L, Leeman L, et al. Validity of self-reported drug use information among pregnant women. Matern Child Health J 2016;20(01):41-47

35 Lange S, Shield K, Koren G, Rehm J, Popova S. A comparison of the prevalence of prenatal alcohol exposure obtained via maternal self-reports versus meconium testing: a systematic literature review and meta-analysis. BMC Pregnancy Childbirth 2014;14 (01):127. Doi: 10.1186/1471-2393-14-127

36 Yazdy MM, Mitchell AA, Tinker SC, Parker SE, Werler MM. Periconceptional use of opioids and the risk of neural tube defects. Obstet Gynecol 2013;122(04):838-844

37 Broussard CS, Rasmussen SA, Reefhuis JNational Birth Defects Prevention Study., et al; . Maternal treatment with opioid analgesics and risk for birth defects. Am J Obstet Gynecol 2011; 204(04):314.e1-314.e11

38 Saia KA, Schiff D, Wachman EM, et al. Caring for pregnant women with opioid use disorder in the USA: expanding and improving treatment. Curr Obstet Gynecol Rep 2016;5(03):257-263

39 Jones HE, O'Grady KE, Malfi D, Tuten M. Methadone maintenance vs. methadone taper during pregnancy: maternal and neonatal outcomes. Am J Addict 2008;17(05):372-386

40 Wright TE, Terplan M, Ondersma SJ, et al. The role of screening, brief intervention, and referral to treatment in the perinatal period. Am J Obstet Gynecol 2016;215(05):539-547

41 Zedler BK, Mann AL, Kim MM, et al. Buprenorphine compared with methadone to treat pregnant women with opioid use disorder: a systematic review and meta-analysis of safety in the mother, fetus and child. Addiction 2016;111(12):2115-2128

42 Substance Abuse and Mental Health Services Administration. A Collaborative Approach to the Treatment of Pregnant Women with Opioid Use Disorders. HHS Publication No. (SMA) 16-4978. Rockville, MD: Substance Abuse and Mental Health Services Administration, 2016. Available at:http://store.samhsa.gov/

43 Krans EE, Kim JY, James AE III, Kelley D, Jarlenski MP. Medicationassisted treatment use among pregnant women with opioid use disorder. Obstet Gynecol 2019;133(05):943-951

44 Martin CE, Longinaker N, Terplan M. Recent trends in treatment admissions for prescription opioid abuse during pregnancy. J Subst Abuse Treat 2015;48(01):37-42

45 Substance Abuse Reporting and Pregnancy: The Role of the Obstetrician-Gynecologist | ACOG. https://www.acog.org/clinical/clinical-guidance/committee-opinion/articles/2011/01/substance-abuse-reporting-and-pregnancy-the-role-of-the-obstetrician-gynecologist. Accessed May 17, 2020

46 Alcohol Abuse and Other Substance Use Disorders: Ethical Issues in Obstetric and Gynecologic Practice | ACOG. https://www.acog.org/ clinical/clinical-guidance/committee-opinion/articles/2015/06/alcohol-abuse-and-other-substance-use-disorders-ethical-issuesin-obstetric-and-gynecologic-practice. Accessed May 17, 2020

47 Roberts SCM, Nuru-Jeter A. Women's perspectives on screening for alcohol and drug use in prenatal care. Womens Health Issues 2010;20(03):193-200

48 Substance Use During Pregnancy | Guttmacher Institute. https:// www.guttmacher.org/state-policy/explore/substance-use-during-pregnancy\#. Accessed May 17, 2020

49 Fitzsimons HE, Tuten M, Vaidya V, Jones HE. Mood disorders affect drug treatment success of drug-dependent pregnant women. J Subst Abuse Treat 2007;32(01):19-25

50 Campus F. 2018Clinical guidance for treating pregnant and parenting women with opioid use disorder and their infantshttps:// content.govdelivery.com/attachments/WADOH/2018/06/13/file_attachments/1023319/SAMHSA_WA_PPW\%2BCME\%2BTraining\%2BFlyer.pdf. Accessed July 20, 2020

51 Krans EE, Bobby S, England M, et al. The Pregnancy Recovery Center: a women-centered treatment program for pregnant and postpartum women with opioid use disorder. Addict Behav 2018; $86: 124-129$ 